\title{
Liver Stiffness-Spleen Size-to-Platelet Ratio Risk Score Identifies Esophageal Varices in Japanese Patients with Chronic Hepatitis C
}

Running title: Identification of esophageal varices in $\mathrm{CHC}$

Soichiro Shibata, Takeji Umemura, Tomoo Yamazaki, Naoyuki Fujimori,

Yuki Ichikawa, Takefumi Kimura, Satoru Joshita, Michiharu Komatsu, Akihiro Matsumoto, Eiji Tanaka

Department of Medicine, Division of Hepatology and Gastroenterology, Shinshu University School of Medicine, Matsumoto, Japan

Corresponding Author: Takeji Umemura, MD, PhD, Department of Medicine, Shinshu University School of Medicine, 3-1-1 Asahi, Matsumoto 390-8621, Japan

E-mail: tumemura@shinshu-u.ac.jp; Tel: +81-263-37-2634; Fax: $+81-263-32-9412$

Conflict of interest: None to declare for any author.

Financial Support: None

Electronic Word Count: 2,050

Number of Figures: 1

Number of Tables: 3 


\section{ABSTRACT}

Aim: Noninvasive methods are needed to identify esophageal varices (EV) in patients with chronic liver disease. To this end, we evaluated liver stiffness (LS)-spleen diameter-to-platelet ratio risk score (LSPS) in predicting EV among Japanese chronic hepatitis C patients.

Methods: A total of 99 patients with chronic hepatitis $\mathrm{C}$ who had undergone endoscopy, LS measurement, and ultrasonography between 2012 and 2014 were enrolled. Clinical data were compared with those for other noninvasive markers (platelet count, aspartate aminotransferase-to-platelet ratio, FIB-4 index, and platelet-to-spleen ratio), spleen size, LS, and controlled attenuation parameter. Diagnostic applicability was assessed by the area under the receiver operating characteristic curve and predictive values along with multivariate logistic regression.

Results: LSPS was significantly correlated to the grade of EV (rho $=0.617, P<$ 0.001) and was superior to the other noninvasive indices for determination of EV. LSPS was independently associated with EV by multivariate logistic regression analysis (odds ratio: 3.079; 95\% confidence interval [Cl]: 2.137-4.438, $P<$ 0.001). The cutoff value of LSPS for EV was 0.7 , for which the area under the receiver operating characteristic curve, sensitivity, specificity, positive predictive value, negative predictive value, and accuracy were $0.928(95 \% \mathrm{Cl}$ : 0.876-0.980), $86.3 \%, 89.6 \%, 70.4 \%, 95.8 \%$, and $88.9 \%$, respectively.

Conclusions: LSPS may also identify EV in patients with chronic hepatitis $C$ in Japan. The clinical values of LSPS for EV risk merit further validation in larger prospective studies. 
Keywords: Liver stiffness, Transient elastography, Fibroscan, LSPS, Esophageal varices 


\section{INTRODUCTION}

Portal hypertension is one of the complications of chronic liver diseases (CLD). Esophageal varices (EV) are the most relevant porto-systemic collaterals resulting from clinically significant portal hypertension, and the presence of EV correlates with the severity of liver disease. As variceal hemorrhage is the most lethal complication of liver cirrhosis, patients with newly diagnosed cirrhosis in CLD are advised to undergo endoscopic screening for EV. However, endoscopy is an invasive and unpleasant procedure that sometimes requires sedation and carries rare, but serious, complications. Accordingly, several simple, noninvasive, and accurate tests have been developed to identify EV. Transient elastography (TE) is a noninvasive tool that measures liver stiffness (LS) correlating to liver fibrosis stage. While TE also shows potential in the prediction of EV, ${ }^{1-8}$ its role is still under debate. Moreover, the LS-spleen size-to-platelet ratio score (LSPS), which is a combination of 3 simple examination methods (LS, spleen size, and platelet count) has been established to accurately predict EV in patients with compensated cirrhosis. ${ }^{9,10}$ To date, TE and LSPS have not been performed for the estimation of EV in chronic hepatitis $C$ in Japan. The objective of this study was to determine the ability of LSPS in predicting the presence of EV in Japanese patients with chronic hepatitis C.

\section{METHODS}

\section{Subjects}

A total of 99 patients with chronic hepatitis $\mathrm{C}$ virus (HCV) infection who were seen at Shinshu University Hospital (Matsumoto, Japan) between April 
2012 and December 2014 were enrolled in this retrospective, cross-sectional study. Their racial background was uniformly Japanese. Exclusion criteria were a history of variceal bleeding and ascites. The diagnosis of chronic hepatitis $\mathrm{C}$ was based on previously reported criteria ${ }^{11}$ as: 1) the presence of serum HCV antibodies and detectable HCV RNA; 2) the absence of detectable hepatitis B surface antigen and antibody to human immune deficiency virus; and 3 ) exclusion of other cases of CLD. This study was conducted in accordance with the principles of the 1975 Declaration of Helsinki. The protocol was reviewed and approved by the institutional ethics committee. Informed consent was obtained from all participants.

\section{Laboratory Testing}

All laboratory data were obtained on the same day as TE scanning. Alanine aminotransferase (ALT), aspartate aminotransferase (AST), alkaline phosphatase, $\mathrm{Y}$-glutamyltransferase, and other relevant biochemical tests were performed using standard methods. Four additional surrogate blood indices of liver fibrosis and EV prediction were also assessed at enrollment according to published formulas: AST-to-platelet ratio index (APRI), ${ }^{12}$ FIB-4 index, ${ }^{13}$ platelet-to-spleen ratio, ${ }^{14}$ and LSPS. ${ }^{9,} 10$

\section{Ultrasonography}

Ultrasonography (US) and TE were performed on the same day following overnight fasting. Spleen size was measured on ultrasound images before TE as spleen bipolar diameter (i.e., crossing the spleen hilium) with a 
convex probe. The experienced US operators were unaware of the clinical details of the patients.

\section{Transient Elastography and Controlled Attenuation Parameter}

TE and controlled attenuation parameter (CAP) were determined with a FibroScan device (Echosens, Paris, France). All patients were assessed using a 3.5 $\mathrm{MHz}$ standard $\mathrm{M}$ probe. Details of the examination procedure have been previously described. ${ }^{15}$ LS was determined as the median value of 10 measurements and expressed in kilopascals $(\mathrm{kPa})$. Results were deemed reliable only when 10 successful acquisitions, a success rate of more than $60 \%$, and a ratio of interquartile range (IQR) to median of less than $30 \%$ were achieved. CAP measurement details have been described previously. ${ }^{16}$ CAP was computed only when the associated LS measurement was valid using the same signal as the one used to measure liver stiffness. Final CAP values were determined as the median of individual CAP values and expressed in $\mathrm{dB} / \mathrm{m}$.

\section{Endoscopy for Esophageal Varices}

Two experienced endoscopists who were unaware of the LS results performed all endoscopic examinations. EV staging was classified as none (no veins above the esophageal mucosal surface; F0), small (minimally elevated veins above the esophageal mucosal surface; F1), medium (large tortuous veins occupying $<1 / 3$ of the lumen; F2), or large (large coil-shaped veins occupying $\geq 1 / 3$ of lumen; F3)..$^{17}$ The high EV risk group included patients with F2/F3 EV or those having F1 EV with red color signs. 


\section{Statistical Analysis}

Categorical variables were compared using the chi-squared or Fisher's exact test, as appropriate, and continuous variables were compared with the Mann-Whitney $U$ test. Multivariate forward stepwise logistic regression analysis was performed to determine the independent factors predicting the absence or presence of EV. Spearman's rank order correlations were used to evaluate the grade of EV of the noninvasive markers. The diagnostic performance of each test for the presence of EV was evaluated by receiver operating characteristic (ROC) curves in terms of sensitivity, specificity, positive predictive value (PPV), negative predictive value (NPV), and area under the ROC curve (AUC). Optimal cutoff values were determined by the Youden index. Comparisons of paired AUCs and $95 \%$ confidence intervals (Cls) were carried out using the nonparametric Delong test. A $P<0.05$ was considered to be statistically significant. Statistical analyses were performed using IBM SPSS Statistics 21.0 (IBM, Chicago, IL) and StatFlex ver. 6.0 (Artech Co., Ltd. Osaka, Japan) software.

\section{RESULTS}

\section{Baseline Clinical Characteristics of Patients}

Of a total of 100 patients examined, one patient with unreliable data was excluded from the analysis. The clinical and biochemical parameters of the remaining 99 patients are listed in Table 1. Median age was 71 years and male incidence was $43 \%$. Seventy-seven patients had no EV, 20 had F1 (1 with red 
color signs), 2 had F2 (1 with red color signs) and none had F3 and red color signs. Overall, 19 patients belonged to the low EV risk group and 3 belonged to the high EV risk group.

\section{Diagnostic Performance of Noninvasive Methods for Esophageal Varices}

In univariate analysis, patients with EV had a significantly older age (78 vs. 70 years, $P<0.001)$, lower platelet count (94 vs. $\left.159 \times 10^{9} / \mathrm{L}, P=0.015\right)$, higher AST level (44 vs. $27 \mathrm{IU} / \mathrm{L}, P=0.024)$, lower albumin level $(3.7 \mathrm{vs.} 4.2 \mathrm{~g} / \mathrm{dL}$, $P<0.001$ ), lower PT\% (83 vs. 96\%, $P=0.008$ ), higher LS (15.8 vs. $5.6 \mathrm{kPa}, P<$ 0.001), lower CAP (182 vs. $207 \mathrm{~dB} / \mathrm{m}, P=0.040$ ), and larger spleen size (10.1 vs. $8.6 \mathrm{~cm}, P=0.039$ ) as compared with patients without EV (Table 1). Moreover, all noninvasive markers (APRI: 1.6 vs. $0.5, P=0.025$; FIB-4: 5.8 vs. $2.5, P<$ 0.001 ; platelet-to-spleen ratio: 879 vs. $1916, P<0.001$; and LSPS: 1.7 vs. $0.3, P$ $<0.001$ ) were significantly associated with EV (Table 1). Multivariate analysis showed that LSPS (odds ratio [OR]: 3.079; 95\% Cl: 2.137-4.438, $P<0.001$ ) and CAP (OR: $0.987 ; 95 \% \mathrm{Cl}: 0.981-0.994, P<0.001$ ) were independently associated with the presence of EV.

Noninvasive markers were next examined for their correlation with the grade of EV (i.e., no EV, low EV risk, and high EV risk) (Table 2). EV grade was significantly correlated with LS, spleen size, APRI, FIB-4 index, and LSPS, and was inversely correlated with platelet count, CAP, and platelet-to-spleen ratio.

$\mathrm{ROC}$ curve analysis was performed to determine the predictive accuracy of LS, LSPS, and other noninvasive parameters in CLD. The values of AUC, optimal cutoff value, sensitivity, specificity, PPV, NPV, and accuracy for the 
presence of EV are listed in Table 3. AUC values were 0.742 for spleen size (95\% Cl: $0.624-0.860, P<0.001), 0.882$ for FIB-4 (95\% Cl: $0.814-0.949, P<$ 0.001), 0.885 for APRI (95\% Cl: 0.821-0.948, $P<0.001), 0.891$ for platelet-to-spleen ratio (95\% Cl: $0.820-0.963, P<0.001), 0.894$ for LS (95\% Cl: $0.825-0.964, P<0.001), 0.896$ for platelet count $(95 \% \mathrm{Cl}: 0.826-0.966, P<$ $0.001)$, and 0.928 for LSPS (95\% Cl: $0.876-0.980, P<0.001)$ (Figure 1$)$. LSPS had the best discrimination for EV, and the AUC of LSPS was significantly superior to that of CAP $(P<0.001$, DeLong test). There were no significant differences between the AUC for LSPS and those for spleen size, FIB-4, LS, APRI, platelet count, or platelet-to-spleen ratio. A cutoff value of LSPS 0.7 had a sensitivity of $86.3 \%$, specificity of $89.6 \%$, PPV of $70.4 \%$, and NPV of $95.8 \%$ (Table 3). In patients with LSPS $<0.7$, the absence of EV was correctly identified with high accuracy $(88.9 \%)$.

\section{DISCUSSION}

TE has been established for the assessment of liver fibrosis, and especially for predicting cirrhosis. Although LS determined by TE was also evaluated for estimation of $\mathrm{EV}$ in cirrhosis, the results were less satisfactory. Recent studies have shown LSPS to be a good predictive marker of EV or high EV risk. ${ }^{9,} 10$ The present investigation therefore explored the abilities of TE, LSPS, and other noninvasive methods (APRI, FIB-4 index, platelet count, platelet-to-spleen ratio, and spleen size) to identify EV in Japanese patients with chronic hepatitis C.

Our results showed that FIB-4 index, APRI, platelet-to-spleen ratio, LS, 
platelet count, and LSPS possessed high AUC values $(>0.880)$ in the prediction of EV. Diagnostic accuracy ranged from $79.8 \%$ to $88.9 \%$ for the presence of EV, with a disease prevalence of $22 \%$. In particular, multivariate and ROC curve analyses indicated that LSPS was the best marker for discriminating EV in our cohort. An LSPS cutoff of $<0.7$ had a $95.8 \%$ NPV for the presence of EV, suggesting a potential role for LSPS in selecting patients for further endoscopic screening. Hence, LSPS $\geq 0.7$ might be a good surrogate marker to identify EV during the surveillance of patients with chronic hepatitis C.

Interestingly, this study demonstrated a negative association between CAP and grade of EV. Moreover, a lower CAP was significantly and independently associated with EV in multivariate analysis. Although CAP was well correlated with the severity of steatosis in chronic hepatitis $C$ in an earlier investigation, ${ }^{18}$ no associations between CAP and EV were reported. As liver histology data were not available for this study, the mechanism of the interesting association between the degree of steatosis and CAP remains undefined.

Hemorrhage of EV often results in more serious subsequent clinical conditions that contribute to higher mortality in CLD. Accordingly, periodic endoscopic screening for EV and appropriate prophylactic treatment for high-risk cases of F2/F3 EV or F1 EV with red color signs are recommended for patients with clinically significant portal hypertension. In Japan, only variceal ligation or injection sclerotherapy for the prevention of variceal hemorrhage are available for patients with a high risk of EV since prophylactic treatment with nonselective $\beta$-blockers has not yet been approved. Thus, simple and reliable noninvasive methods are needed to better identify high EV risk prior to endoscopy. The 
diagnostic accuracy of LSPS as determined by the AUC in the present study was similar to that of published data, ${ }^{9,} 10$ albeit at different thresholds; Kim et al. ${ }^{9}$ reported a lower cutoff of 3.5 (sensitivity: $70.0 \%$, specificity: $82.9 \%$, PPV: $50.0 \%$, and NPV: $91.9 \%$ ) and higher cutoff of 5.5 (sensitivity: 50.0\%, specificity: $92.7 \%$, PPV: $62.5 \%$, and NPV: $88.4 \%$ ) in patients with HBV-related cirrhosis. Although median LSPS tended to be higher in the high risk group than in the low risk group (2.8 vs. $1.7, P=0.087$ ), only 3 patients had high $\mathrm{EV}$ risk in our cohort, making it difficult to draw any definite conclusions on LSPS and high-risk EV status. Larger studies are needed to assess this possible association after increasing the number of patients with high EV risk.

There are several limitations to the present investigation. First, since it was a cross-sectional retrospective study, the incidence of EV or bleeding EV could not be determined. Second, the relatively small sample size, especially for high EV risk may have influenced the results. Third, although TE is a promising method to estimate liver fibrosis, it is not applicable for patients with ascites or obesity, which precludes the use of LSPS in these individuals. Recently, serum Wisteria floribunda agglutinin-positive Mac-2-binding protein was reported as a novel, noninvasive method of estimating liver fibrosis ${ }^{19}$ that was confirmed in patients with $\mathrm{HCV}, \mathrm{NASH}$, and PBC. ${ }^{20-23}$ Prospective studies on the prediction of EV or high EV risk using Wisteria floribunda agglutinin-positive Mac-2-binding protein and similar surrogate biomarkers may overcome such issues.

In conclusion, we have demonstrated that LSPS is a potent, noninvasive method for predicting EV in Japanese patients with chronic hepatitis C. Future trials are warranted to evaluate this, and other, noninvasive methods for 
discriminating $\mathrm{EV}$ and a high risk of $\mathrm{EV}$ in a prospective setting.

\section{ACKNOWLEDGMENTS}

The authors thank Mie Karakida for her secretarial assistance and Trevor Ralph for his English editorial assistance. 


\section{References}

$1 \quad$ Kazemi F, Kettaneh A, N'Kontchou G, et al. Liver stiffness measurement selects patients with cirrhosis at risk of bearing large oesophageal varices. J Hepatol 2006;45: 230-5.

$2 \quad$ Vizzutti F, Arena U, Romanelli RG, et al. Liver stiffness measurement predicts severe portal hypertension in patients with HCV-related cirrhosis. Hepatology 2007;45: 1290-7.

3 Bureau C, Metivier S, Peron JM, et al. Transient elastography accurately predicts presence of significant portal hypertension in patients with chronic liver disease. Aliment Pharmacol Ther 2008;27: 1261-8.

4 Castera L, Le Bail B, Roudot-Thoraval F, et al. Early detection in routine clinical practice of cirrhosis and oesophageal varices in chronic hepatitis $\mathrm{C}$ : comparison of transient elastography (FibroScan) with standard laboratory tests and non-invasive scores. J Hepatol 2009;50: 59-68.

$5 \quad$ Malik R, Lai M, Sadiq A, et al. Comparison of transient elastography, serum markers and clinical signs for the diagnosis of compensated cirrhosis. J Gastroenterol Hepatol 2010;25: $1562-8$

6 Pritchett S, Cardenas A, Manning D, Curry M, Afdhal NH. The optimal cut-off for predicting large oesophageal varices using transient elastography is disease specific. $J$ Viral Hepat 2011;18: e75-80.

7 Chen YP, Zhang Q, Dai L, Liang XE, Peng J, Hou JL. Is transient elastography valuable for high-risk esophageal varices prediction in patients with hepatitis-B-related cirrhosis? $J$ Gastroenterol Hepatol 2012;27: 533-9.

$8 \quad$ Wang JH, Chuah SK, Lu SN, et al. Transient elastography and simple blood markers in the diagnosis of esophageal varices for compensated patients with hepatitis $B$ virus-related cirrhosis. J Gastroenterol Hepatol 2012;27: 1213-8.

9 Kim BK, Han KH, Park JY, et al. A liver stiffness measurement-based, noninvasive prediction model for high-risk esophageal varices in B-viral liver cirrhosis. Am J Gastroenterol 2010;105: 1382-90.

10 Berzigotti A, Seijo S, Arena U, et al. Elastography, spleen size, and platelet count identify portal hypertension in patients with compensated cirrhosis. Gastroenterology 2013;144: 102-11 e1.

11 Umemura T, Wang RY, Schechterly C, Shih JW, Kiyosawa K, Alter HJ. Quantitative analysis of anti-hepatitis $C$ virus antibody-secreting $B$ cells in patients with chronic hepatitis $C$. Hepatology 2006;43: 91-9.

12 Wai CT, Greenson JK, Fontana RJ, et al. A simple noninvasive index can predict both significant fibrosis and cirrhosis in patients with chronic hepatitis C. Hepatology 2003;38: 518-26. 13 Sterling RK, Lissen E, Clumeck N, et al. Development of a simple noninvasive index to predict significant fibrosis in patients with HIV/HCV coinfection. Hepatology 2006;43: 1317-25.

14 Giannini E, Botta F, Borro P, et al. Platelet count/spleen diameter ratio: proposal and validation of a non-invasive parameter to predict the presence of oesophageal varices in patients with liver cirrhosis. Gut 2003;52: 1200-5.

15 Sandrin L, Fourquet B, Hasquenoph JM, et al. Transient elastography: a new noninvasive method for assessment of hepatic fibrosis. Ultrasound Med Biol 2003;29: 1705-13.

16 Sasso $\mathrm{M}$, Beaugrand $\mathrm{M}$, de Ledinghen $\mathrm{V}$, et al. Controlled attenuation parameter (CAP): a novel VCTE guided ultrasonic attenuation measurement for the evaluation of hepatic steatosis: preliminary study and validation in a cohort of patients with chronic liver disease from various causes. Ultrasound Med Biol 2010;36: 1825-35.

17 Beppu K, Inokuchi K, Koyanagi N, et al. Prediction of variceal hemorrhage by esophageal endoscopy. Gastrointest Endosc 1981;27: 213-8.

18 Calvaruso V, Bronte F, Conte E, Simone F, Craxi A, Di Marco V. Modified spleen stiffness measurement by transient elastography is associated with presence of large oesophageal varices in patients with compensated hepatitis $\mathrm{C}$ virus cirrhosis. $J$ Viral Hepat 2013;20: 867-74.

19 Kuno A, Ikehara Y, Tanaka Y, et al. A serum "sweet-doughnut" protein facilitates fibrosis evaluation and therapy assessment in patients with viral hepatitis. Sci Rep 2013;3: 1065.

20 Toshima T, Shirabe K, Ikegami T, et al. A novel serum marker, glycosylated Wisteria floribunda agglutinin-positive Mac-2 binding protein (WFA-M2BP), for assessing liver fibrosis. $J$ Gastroenterol 2014;50: 76-84. 
21 Yamasaki K, Tateyama M, Abiru S, et al. Elevated serum levels of Wisteria floribunda agglutinin-positive human Mac-2 binding protein predict the development of hepatocellular carcinoma in hepatitis C patients. Hepatology 2014;60: 1563-70.

22 Abe M, Miyake T, Kuno A, et al. Association between Wisteria floribunda agglutinin-positive Mac-2 binding protein and the fibrosis stage of non-alcoholic fatty liver disease. J Gastroenterol 2014.

23 Umemura T, Joshita S, Sekiguchi T, et al. Serum Wisteria floribunda Agglutinin-Positive Mac-2-Binding Protein Level Predicts Liver Fibrosis and Prognosis in Primary Biliary Cirrhosis. Am J Gastroenterol 2015;110: 857-64. 


\section{Figure Legend}

Figure 1. Receiver operating characteristic curve of LSPS for the identification of esophgeal varices. 
Table 1. Clinical variables of patients with and without esophageal varices

\begin{tabular}{|c|c|c|c|c|c|c|c|}
\hline \multirow{2}{*}{$\begin{array}{l}\text { Variable } \\
\text { Age (years) }\end{array}$} & \multicolumn{2}{|c|}{ Total $(n=99)$} & \multicolumn{2}{|c|}{$E V(+)(n=22)$} & \multicolumn{2}{|c|}{$\operatorname{EV}(-)(n=77)$} & \multirow{2}{*}{$\frac{P \text { value }}{<0.001}$} \\
\hline & 71 & $(62-77)$ & 78 & $(74-81)$ & 70 & $(62-75)$ & \\
\hline Male gender, n (\%) & 43 & (43) & 11 & (50) & 32 & (42) & 0.481 \\
\hline BMI & 21.8 & $(20.1-23.8)$ & 21.7 & $(20.2-23.6)$ & 22.0 & $(20.0-23.1)$ & 0.161 \\
\hline HCC, n (\%) & 12 & (12) & 8 & (36) & 4 & (5) & $<0.001$ \\
\hline Platelet count $\left(\times 10^{9} / \mathrm{L}\right)$ & 151 & $(110-184)$ & 94 & $(74-105)$ & 159 & $(141-200)$ & 0.015 \\
\hline AST (IU/L) & 30 & $(22-40)$ & 44 & $(33-49)$ & 27 & $(21-34)$ & 0.024 \\
\hline ALT (IU/L) & 22 & $(18-33)$ & 33 & $(28-48)$ & 20 & $(17-28)$ & 0.059 \\
\hline Albumin (g/dl) & 4.2 & $(3.9-4.4)$ & 3.7 & $(3.3-4.0)$ & 4.2 & $(4.0-4.5)$ & $<0.001$ \\
\hline T-Bil (mg/dl) & 0.8 & $(0.7-1.0)$ & 0.8 & $(0.7-1.5)$ & 0.8 & $(0.7-0.9)$ & 0.117 \\
\hline РT\% & 92 & $(82-103)$ & 83 & $(76-87)$ & 96 & $(85-105)$ & 0.008 \\
\hline LS (kPa) & 6.2 & $(4.5-10.2)$ & 15.8 & $(10.0-25.1)$ & 5.6 & $(4.1-7.0)$ & $<0.001$ \\
\hline $\mathrm{CAP}(\mathrm{dB} / \mathrm{m})$ & 205 & $(167-232)$ & 182 & $(162-210)$ & 207 & $(173-241)$ & 0.040 \\
\hline Spleen size $(\mathrm{cm})$ & 8.9 & $(7.8-10.1)$ & 10.1 & $(8.9-11.0)$ & 8.6 & $(7.7-9.6)$ & 0.039 \\
\hline LSPS & 0.4 & $(0.2-0.9)$ & 1.7 & $(1.3-2.8)$ & 0.3 & $(0.2-0.4)$ & $<0.001$ \\
\hline APRI & 0.6 & $(0.4-1.3)$ & 1.6 & $(1.1-2.6)$ & 0.5 & $(0.4-0.7)$ & 0.025 \\
\hline FIB-4 & 2.8 & $(1.9-4.1)$ & 5.8 & $(3.9-10.7)$ & 2.5 & $(1.9-3.4)$ & $<0.001$ \\
\hline Platelet-to-spleen ratio & 1700 & $(1091-2345)$ & 879 & $(692-1120)$ & 1916 & $(1540-2523)$ & $<0.001$ \\
\hline
\end{tabular}


Table 2. Correlation between noninvasive markers and grade of esophageal varices in 99 patients with chronic hepatitis C

\begin{tabular}{lcc}
\hline Characteristic & rho & $\boldsymbol{P}$ value \\
\hline Platelet count $\left(\times 10^{9} / \mathrm{L}\right)$ & -0.571 & $<0.001$ \\
LS $(\mathrm{kPa})$ & 0.569 & $<0.001$ \\
CAP $(\mathrm{dB} / \mathrm{m})$ & -0.204 & 0.043 \\
Spleen size & 0.348 & $<0.001$ \\
LSPS & 0.617 & $<0.001$ \\
APRI & 0.557 & $<0.001$ \\
FIB-4 index & 0.551 & $<0.001$ \\
Platelet-to-spleen ratio & -0.564 & $<0.001$ \\
\hline
\end{tabular}


Table 3. Performance of noninvasive parameters for identifying esophageal varices

\begin{tabular}{|c|c|c|c|c|c|c|c|}
\hline Variable & Cutoff & AUC $(95 \% \mathrm{Cl})$ & Sensitivity (\%) & Specificity (\%) & PPV (\%) & NPV (\%) & Accuracy (\%) \\
\hline $\mathrm{CAP}(\mathrm{dB} / \mathrm{m})$ & 216 & $0.644(0.527-0.762)$ & 86.4 & 40.3 & 29.2 & 91.2 & 50.5 \\
\hline Spleen size (cm) & 8.6 & $0.742(0.624-0.860)$ & 90.9 & 51.9 & 35.1 & 95.2 & 60.6 \\
\hline FIB-4 index & 3.7 & $0.882(0.814-0.949)$ & 81.8 & 81.8 & 56.3 & 94.0 & 81.8 \\
\hline APRI & 0.7 & $0.885(0.821-0.948)$ & 95.5 & 75.3 & 52.5 & 98.3 & 79.8 \\
\hline $\begin{array}{l}\text { Platelet-to-spleen } \\
\text { ratio }\end{array}$ & 1480 & $0.891(0.820-0.963)$ & 86.4 & 79.2 & 20.8 & 54.3 & 80.8 \\
\hline LS $(\mathrm{kPa})$ & 7.9 & $0.894(0.825-0.964)$ & 86.4 & 83.1 & 59.3 & 95.5 & 83.8 \\
\hline $\begin{array}{l}\text { Platelet count } \\
\left.\qquad \times 10^{9} / \mathrm{L}\right)\end{array}$ & 141 & $0.896(0.826-0.966)$ & 95.5 & 75.3 & 52.5 & 98.3 & 79.8 \\
\hline LSPS & 0.7 & $0.928(0.876-0.980)$ & 86.3 & 89.6 & 70.4 & 95.8 & 88.9 \\
\hline
\end{tabular}

